\title{
De-novo emergence of SINE retroposons during the early evolution of passerine birds
}

\author{
Alexander Suh ${ }^{1,2^{*}}$ (D), Sandra Bachg ${ }^{1}$, Stephen Donnellan ${ }^{3,4}$, Leo Joseph ${ }^{5}$, Jürgen Brosius ${ }^{1,6}$, Jan Ole Kriegs ${ }^{1,7}$ \\ and Jürgen Schmitz ${ }^{1}$
}

\begin{abstract}
Background: Passeriformes ("perching birds" or passerines) make up more than half of all extant bird species. The genome of the zebra finch, a passerine model organism for vocal learning, was noted previously to contain thousands of short interspersed elements (SINEs), a group of retroposons that is abundant in mammalian genomes but considered largely inactive in avian genomes.

Results: Here we resolve the deep phylogenetic relationships of passerines using presence/absence patterns of SINEs. The resultant retroposon-based phylogeny provides a powerful and independent corroboration of previous sequence-based analyses. Notably, SINE activity began in the common ancestor of Eupasseres (passerines excluding the New Zealand wrens Acanthisittidae) and ceased before the rapid diversification of oscine passerines (suborder Passeri - songbirds). Furthermore, we find evidence for very recent SINE activity within suboscine passerines (suborder Tyranni), following the emergence of a SINE via acquisition of a different tRNA head as we suggest through template switching.

Conclusions: We propose that the early evolution of passerines was unusual among birds in that it was accompanied by de-novo emergence and activity of SINEs. Their genomic and transcriptomic impact warrants further study in the light of the massive diversification of passerines.
\end{abstract}

Keywords: Transposon, Retroposon, SINE, Birds, Passeriformes, Phylogenomics

\section{Background}

Short interspersed elements (SINEs) are the most abundant group of the reverse-transcribed retroposons in mammalian genomes [1]. They rely on trans-mobilization by the enzymatic machinery of long interspersed elements (LINEs) [2], a parasitic interaction so successful that the human genome contains $>1,500,000$ SINEs compared to $<900,000$ LINEs [3]. On the other hand, SINEs are scarce in avian genomes, and this has been noted as one of the most peculiar genomic features of birds [4-6]. While LINEs exhibit up to 700,000 copies in avian genomes,

\footnotetext{
* Correspondence: alexander.suh@ebc.uu.se

${ }^{1}$ Institute of Experimental Pathology (ZMBE), University of Münster, D-48149 Münster, Germany

${ }^{2}$ Department of Evolutionary Biology (EBC), Uppsala University, SE-75236

Uppsala, Sweden

Full list of author information is available at the end of the article
}

there are only 6000-17,000 SINEs per avian genome [6], most of these being ancient and heavily degraded [7].

Presence/absence patterns of SINEs in orthologous genomic loci are rare genomic changes appreciated widely as virtually homoplasy-free phylogenetic markers $[8,9]$. Given the aforementioned scarcity of SINEs, it is not surprising that the emergence and activity of SINEs has never been studied in birds. On the other hand, other types of retroposed elements (REs; LINEs from the chicken repeat 1 superfamily, CR1, and long terminal repeat elements, LTRs) have helped resolve the relationships of various groups of birds, such as Galliformes [10-12], Neoaves [13-15], Palaeognathae [16, 17], and others [18-21]. In the meantime, the sequencing of dozens of avian genomes has revealed SINEs with putative lineage specificity $[5,7,22]$ and thus the potential 
for conducting phylogenetic presence/absence analyses in specific groups of birds.

Here we conduct, to our knowledge, the first study of the emergence and activity of SINEs in birds. We focus on the deep phylogenetic relationships of passerines, the largest radiation of birds with nearly 6000 extant species [23], using 44 presence/absence markers of SINEs and other REs. In contrast to the only previous study of retroposons in passerines with a single RE marker [24], our multilocus dataset permits the reassessment of sequence-based phylogenies (e.g., [23, 25, 26]) and, simultaneously, the reconstruction of the temporal activity of SINEs and other REs during early passerine evolution.

\section{Results and discussion}

\section{Two CR1-mobilized SINEs in passerines}

We initially chose RE marker candidates from selected retroposon families of the oscine passerine zebra finch Taeniopygia guttata (including TguSINE1, [5]; Additional file 1: Table S1) in October 2009, a time when genome assemblies were available only for chicken and zebra finch $[4,5]$. Seventy four candidates for presence/absence loci were therefore identified via pairwise alignment of RE-flanking sequences from zebra finch to orthologous regions in chicken (Materials and Methods). This was followed by invitro presence/absence screening of RE marker candidates as detailed elsewhere $[13,27]$ using a representative taxon sampling of all major groups of passerines sensu Barker et al. [23] (Additional file 1: Table S2). We complemented this with a screening of GenBank [28] for additional SINEs, which identified a TguSINE1-like insertion in myoglobin intron 2 of the suboscine Pitta anerythra (accession number DQ785977) that is absent in the orthologous position of other Pitta species [29]. We termed this element "PittSINE" and identified PittSINE marker candidates in a DNA sample of Pitta sordida via inter-SINE PCR ([30]; Methods). This was followed by cloning of the 500-bp to 1000-bp fraction of PCR amplicons and sequencing of 24 clones, alignment to chicken and zebra finch genomes to reconstruct the left and right SINEflanking regions, and then in-vitro presence/absence screening of nine PittSINE marker candidates.

Next, we characterized the structural organization of passerine SINEs (Fig. 1) using the available TguSINE1 consensus sequence [5] and after generating a majorityrule consensus of six PittSINE insertions in our sequenced presence/absence markers (Additional file 2). Both SINEs have highly similar, CR1-derived tails (Fig. 1) which exhibit the typical hairpin for putative binding by the CR1 reverse transcriptase and an 8-bp microsatellite at their very end for target-primed reverse transcription [31] (Additional file 3: Figure S1). However, the heads of these SINEs are derived from different tRNA genes, namely tRNA $^{\text {Ile }}$ in TguSINE1 and tRNA ${ }^{\text {Asp }}$ in
PittSINE (Fig. 1). Sequence alignment suggests that the tRNA-derived SINE heads are more similar to the respective tRNA genes than they are to each other (Fig. 1c). However, the opposite is the case for the CR1derived SINE tails, which exhibit four diagnostic nucleotides distinguishing them from the highly similar $3^{\prime}$ end of CR1-X1_Pass (Fig. 1c). To verify that these are specific to TguSINE1 and PittSINE, we screened the zebra finch genome assembly for the presence of the four diagnostic nucleotides in copies of CR1-X1_Pass. Among those copies most similar to CR1-X1_Pass, only one old copy (chr2:68,921,881-68,922,556) contained the four diagnostic nucleotides, suggesting that these were acquired randomly after the insertion event.

We further investigated this peculiar pattern using phylogenetic analyses of the CR1-derived SINE tails and avian CR1 subfamilies sensu ref. [32], which again suggests that TguSINE1 and PittSINE have a single SINE ancestor which derived its tail from CR1-X1_Pass (Fig. 2a). Assuming that SINEs are trans-mobilized by LINE reverse transcriptase enzymes due to high sequence similarity between SINE tails and LINE 3' ends $[2,33]$ and thus depend on LINE activity, the most likely candidate for SINE mobilization is the CR1-X1_Pass subfamily. This is further supported by temporal overlap of TguSINE1 and CR1-X activity in RE landscapes of the zebra finch genome (Fig. 2b). Additionally, we detected direct evidence for temporal overlap of TguSINE1 and CR1-X1_Pass activity through our presence/absence analyses (Fig. 3a, Additional file 1: Table S2).

\section{Retroposon-based phylogeny of passerines}

Our extensive RE presence/absence analyses yielded 19 TguSINE1, 6 PittSINE, 13 CR1, and 6 LTR markers which we could trace across a representative taxon sampling of the major groups of passerines sensu Barker et al. [23] (cf. [34]). These RE markers are only those where we were able to obtain sequences for all taxa critical for a phylogenetic conclusion. Careful inspection of presence/absence alignments using strict criteria (see Materials and Methods) yielded a conflict-free set of RE markers (except for one marker potentially affected by incomplete lineage sorting; Fig. 3a), which we mapped on a maximum likelihood tree constructed from concatenated RE-flanking sequences from the same data set (Fig. 3a). For three of the deepest passerine branching events, we found a multitude of RE markers and thus statistically significant support in available $R E$ marker tests $[35,36]$. These relationships are the respective monophyly of passerines and oscines, as well as the monophyly of Eupasseres [37], a group comprising all passerines except the New Zealand wrens Acanthisittidae. The Eupasseres/Acanthisittidae split was first observed in sequence analyses of few nuclear genes [38, 


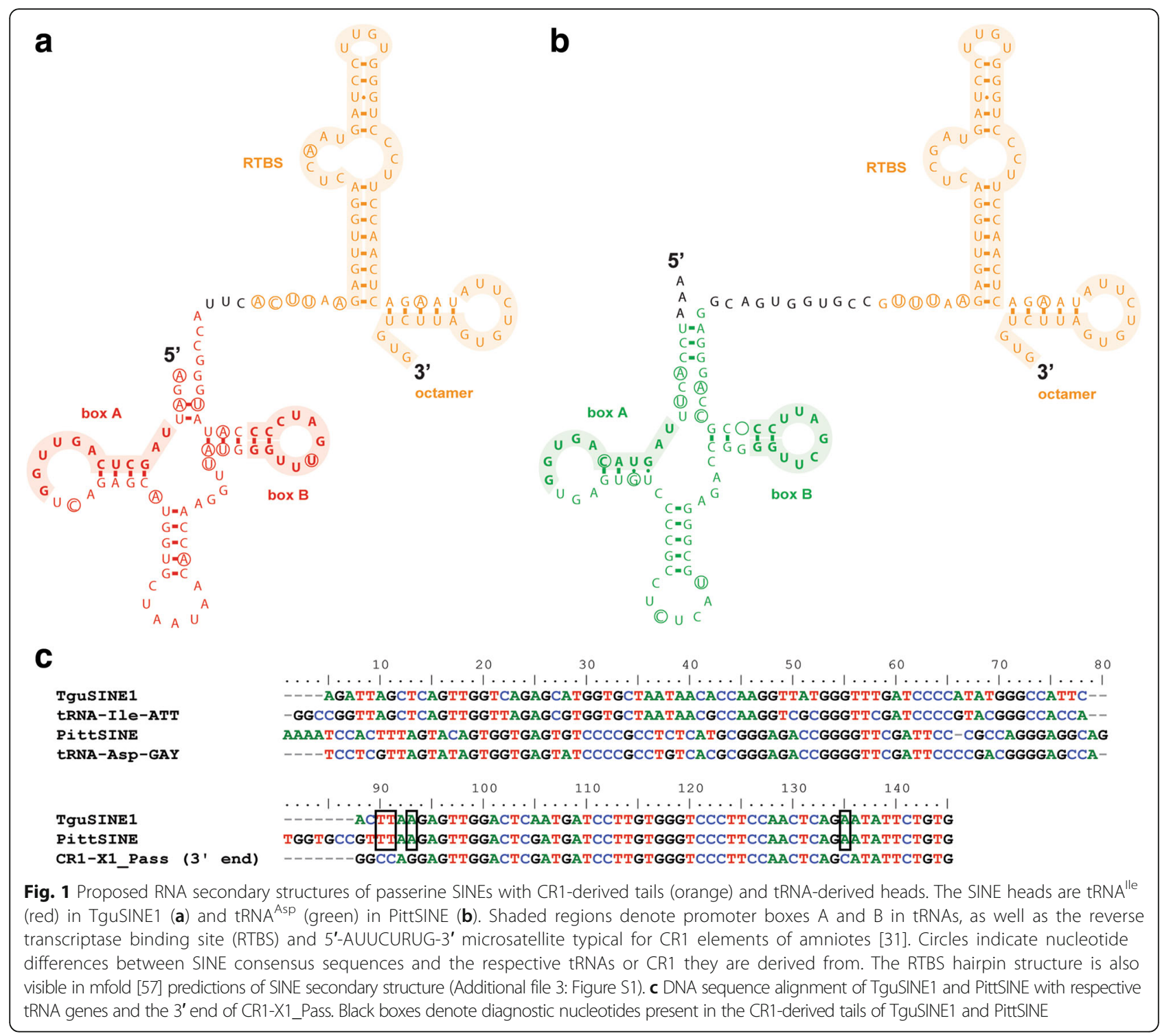

39] and has since been confirmed in ever-growing nuclear sequence analyses (e.g., [23, 25, 26, 40]). Our analysis of rare genomic changes thus provides the first assessment of this group using an independent marker type and phylogenetic method. None of our RE markers inserted during the rapid radiation of oscine passerines, however, sequence analysis of the RE-flanking regions yielded a topology identical to the aforementioned previous studies. Of particular interest are the four deepbranching oscine lineages Menuridae (e.g., Menura novaehollandiae), Climacteridae (e.g., Climacteris picumnus), Maluridae/Meliphagidae (e.g., Malurus cyaneus and Myzomela eques), and Pomatostomidae (e.g., Pomatostomus superciliosus) because these four lineages together have been rarely included in passerine phylogenetic studies. We find a branching order (Fig. 3a) which recapitulates previous phylogenetic estimates based on few nuclear genes [23] or ultraconserved elements [26]. This suggests that the rapid radiation of oscines can be congruently resolved even with nongenome-scale data. We note that this is in contrast to the neoavian radiation, which appears to be partially unresolvable even with genome-scale sequence analyses and thousands of retroposon markers (reviewed by [41]). Within passerines, we further note that the conflict between single-RE support for a Picathartidae/Corvidae clade [24] and sequence-based phylogenies [42] results from incorrect placing of this RE marker on the passerine Tree of Life due to methodological limitations (see legend of Fig. 4 for more information).

\section{Emergence and activity of passerine SINEs}

We then traced the emergence and activity of SINEs across the passerine Tree of Life. Given that RE marker 


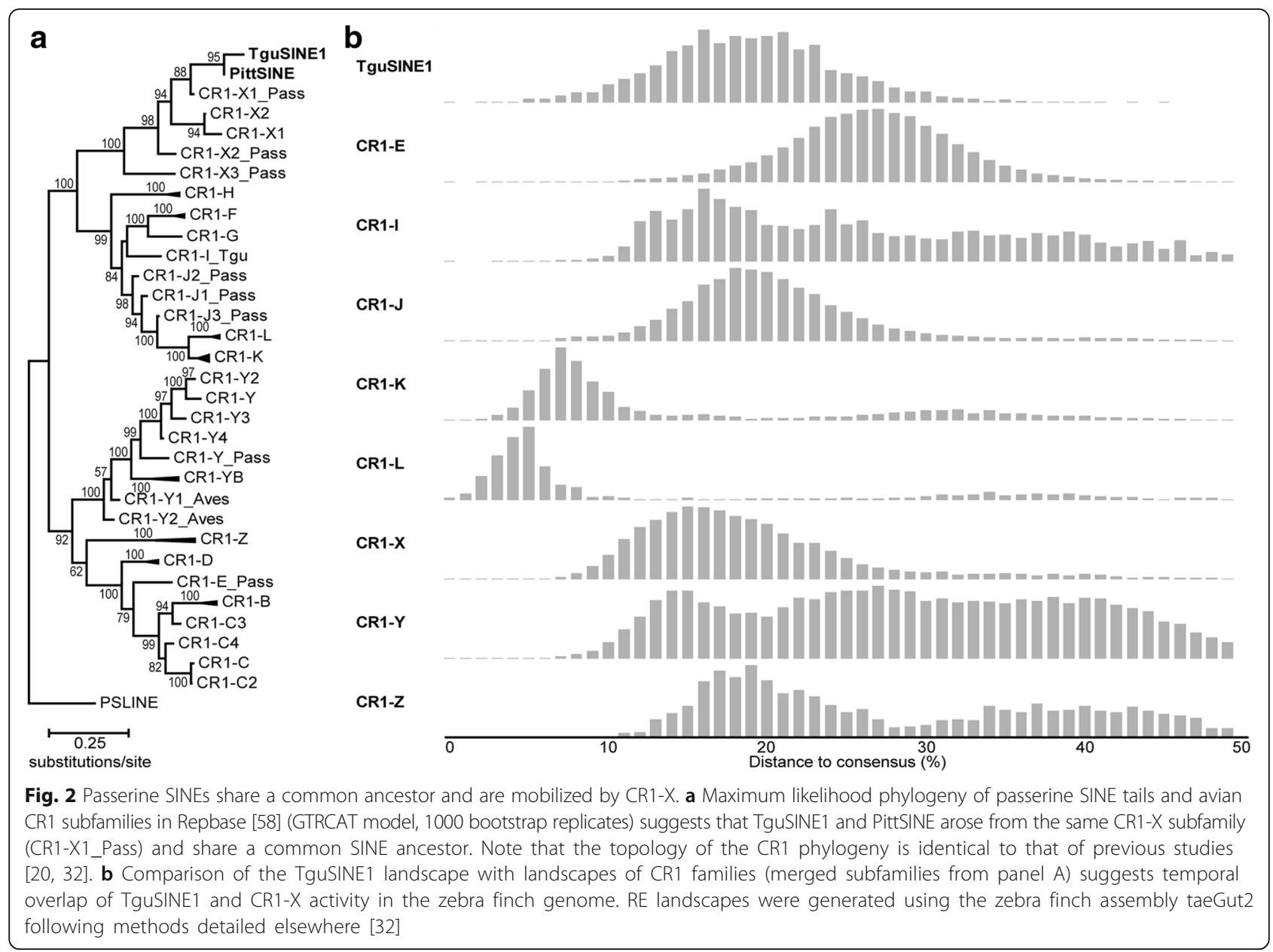

candidates were initially chosen on chicken/zebra finch alignments, we expect no bias in the age distribution of RE markers on the lineage leading to zebra finch. TguSINE1 was mostly active in the ancestor of oscines and, to a lesser extent, in the ancestor of Eupasseres. Interestingly, we find no evidence for TguSINE1 activity in the common ancestor of passerines (cf. Additional file 3: Figure S2) or during/after the radiation of oscines and therefore hypothesize that TguSINE1 emerged in Eupasseres and became extinct in the oscines' ancestor (Fig. 3a). The emergence of TguSINE1 is thus the first synapomorphic "genome morphology" character for Eupasseres and supplements support from skeletal morphology, which is limited to the presence of a 'six-canal pattern' in the hypotarsus [43].

In contrast to the situation in oscines, the activity of TguSINE1 appears to have been longer in suboscines, postdating the divergence between Old World and New World suboscines (i.e., pitta and phoebe in Fig. 3a). This recent, potentially lineage-specific activity coincides with the putative restriction of PittSINEs to Old World suboscines (e.g., Pitta spp.), which is further supported by a much lower pairwise distance of PittSINE copies to the consensus (ranging from 0 to $11 \%$, average 6.3\%; Additional file 1: Table
S3) than in the case of TguSINE1 (Fig. 2b). As mentioned above, the CR1 phylogeny and four diagnostic nucleotides in the CR1-derived SINE tails (cf. Figs. 1c and 2a) indicate that TguSINE1 and PittSINE likely have a common SINE ancestor instead of being derived independently from a CR1-X_Pass LINE. This further suggests that the younger PittSINE emerged from the older TguSINE1 after acquisition of a new tRNA-derived head. Assuming that TguSINE1 and PittSINE were both active on the pitta lineage, we propose that the most plausible mechanism for PittSINE emergence was template switching from TguSINE1 to a nearby tRNA during reverse transcription (Fig. 3b). Slightly less parsimonious alternative explanations for PittSINE emergence might be gene conversion or genomic rearrangement between a TguSINE1 master gene and a tRNA ${ }^{\text {Asp }}$ gene, but these remain untestable in the absence of a pitta genome assembly. Template switching has been previously proposed in a wide range of chimeric retroposons (e.g., [44-47]) and appears to be a particularly common opportunity for SINEs to parasitize different LINEs via acquisition of new SINE tails [46, 48]. As previously observed for ancient amniote SINEs [49], our data show that template switching may also happen for SINE heads, whereby the 


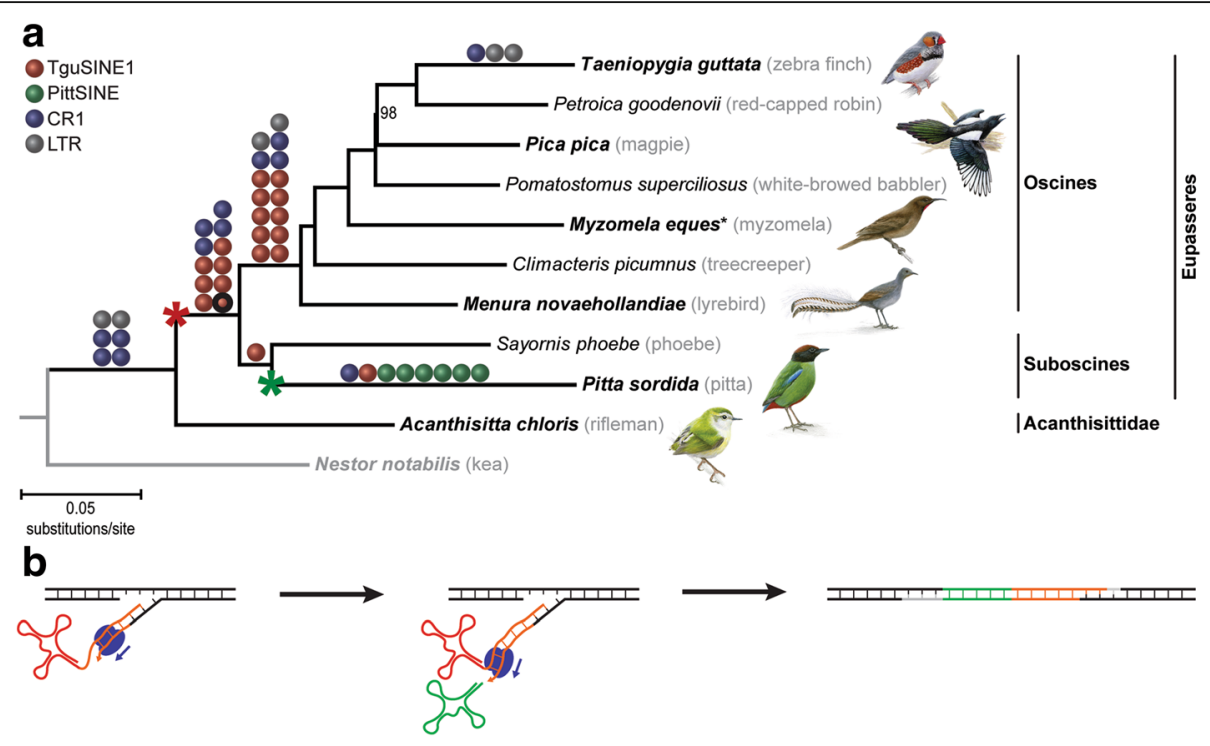

Fig. 3 Emergence and timing of CR1-mobilized SINE activity during early passerine evolution. a Phylogenomic analysis of early passerine relationships using retroposon presence/absence markers (colored balls) mapped on a maximum likelihood phylogeny of concatenated retroposon-flanking sequences (GTRCAT model, 1000 bootstrap replicates; Additional file 5). The single conflicting marker on the Eupasseres branch (Tgu10, cf. Additional file 1: Table S2) is indicated by a red ball with black circle and was likely affected by incomplete lineage sorting within Suboscines. Our sampling consists of the major deep passerine lineages sensu Barker et al. [23]. The later additions of two genome assemblies (Corvus cornix and Manacus vitellinus) were only included in the presence/absence table (Additional file 1: Table S2). Red and green asterisks indicate emergence of TguSINE1 and PittSINE, respectively. The black asterisk indicates that for some loci (Additional file 1: Table S2), Malurus cyaneus was sampled instead of Myzomela eques to represent the Maluridae/Meliphagidae clade [23]. Only bootstrap values $<100 \%$ are shown and the names of pictured birds are emphasized in bold. $\mathbf{b}$ A scenario for the emergence of PittSINE. Template switching from TguSINE1 RNA (red, tRNA ${ }^{\text {lle }}$ head; orange, CR1 tail) to tRNA ${ }^{\text {Asp }}$ (green) during target-primed reverse transcription by CR1 reverse transcriptase (blue). The resultant tRNA ${ }^{\text {Asp }}$-CR1 chimaera was flanked by a target site duplication (grey) and transcriptional activation gave rise to the PittSINE family

acquisition of a new SINE head from a different tRNA and an appropriate upstream sequence close to the insertion site may provide intact and active promoter components for efficient transcription by RNA polymerase III.

\section{Conclusion}

To conclude, we reconstructed the deep phylogenetic relationships of passerines using presence/absence patterns of unusual SINE insertions and other REs. This permitted us to follow the emergence, activity, and extinction of TguSINE1 and PittSINE across the evolution of the most species-rich group of birds. While this SINE activity of $\sim 2000$ copies per oscine genome and $\sim 2500$ copies per suboscine genome (Additional file 3: Figure S2) was considerably lower than, for example, that in mammals, it nevertheless exemplifies that at least some birds have

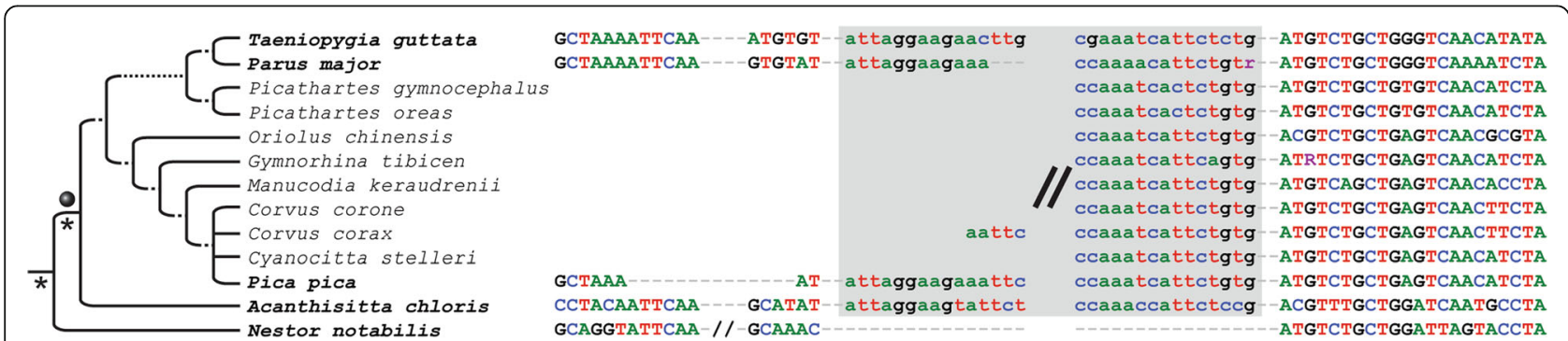

Fig. 4 A reassessment of the RE marker of Treplin \& Tiedemann [24] through extended taxon sampling. Treplin \& Tiedemann [24] inferred "phylogenetic affinity of rockfowls (genus Picathartes) to crows and ravens (Convidae)" based on the Cor2 locus which they sequenced in rockfowls and corvids, and unsuccessful Cor2 PCR amplification in other passerines. We generated a nested oligonucleotide primer pair (5'- CAATACTTTGGAACACCTCAGAC-3' and 5'GGCACCTGTCAATGGCTAC-3') and were able to amplify and sequence the Cor2 locus in additional species. Our extended phylogenetic sampling suggests that the RE insertion (lowercase nucleotides) occurred in the ancestor of all passerines (grey ball) due to RE presence in non-corvid passerines (Taeniopygia guttata, Parus major, Acanthisitta chloris) and RE absence in the parrot outgroup (Nestor notabilis). Taxa with bold names were sampled in the present study and the grey box denotes the $5^{\prime}$ and $3^{\prime}$ end of the CR1 insertion. Asterisks indicate branches in the avian Tree of Life which were previously recovered with significant support from retroposon markers [13] 
a more diverse repetitive element landscape than previously anticipated. Furthermore, we note that the activity of TguSINE1 appears to coincide with the evolution of vocal learning during early passerine evolution [13]. Previous evidence suggests that $\sim 4 \%$ of birdsong-associated transcripts in the zebra finch brain contain retroposons [5] and it thus remains to be seen whether SINE activity influenced the evolution of, for example, vocal learning in oscine passerines.

\section{Methods}

We identified candidates for presence/absence loci for TguSINE1 and other selected zebra finch retroposons via pairwise alignment of RE loci from zebra finch to orthologous regions in chicken. This was done by comparing and extracting the respective RE-flanking sequences in the UCSC Genome Browser [50], followed by automatic alignment using MAFFT version 6 [51]. In order to find the nine PittSINE marker candidates, we conducted interSINE PCR [30] using a single, PittSINE-specific oligonucleotide primer (5'-CTCGTTAGTATAGTGGTGAGTGTC-3') and standard PCR parameters of ref. [27] with $50{ }^{\circ} \mathrm{C}$ annealing temperature. Among the sampled passerines, inter-SINE PCR yielded strong amplification signal only in the pitta (data not shown). Additionally, we identified two TguSINE1 candidate loci in the pitta using a single TguSINE1-specific oligonucleotide primer (5'CAGTTGGTTAGAGCGTGGTG-3'). All presence/absence screenings were done using oligonucleotide primers binding to conserved RE-flanking regions in chicken/zebra finch alignments (Additional file 1: Table S4), using the touchdown PCR and cloning protocols of ref. [13]. Two recently sequenced species (Corvus cornix and Manacus vitellinus $[6,52])$ were added to reduce missing data in our presence/absence table (Additional file 1: Table S2).

For each presence/absence marker candidate, we first aligned all sequences automatically using MAFFT (EINS-I option) and then manually inspected these for misalignments. We considered a marker candidate as phylogenetically informative and reliable "if, in all species sharing this $R E$, it featured an identical orthologous genomic insertion point (target site), identical RE orientation, identical RE subtype, identical target site duplications (direct repeats, if present) and a clear absence in other species" [13]. This led to a total of 44 highquality RE presence/absence markers (Additional file 1: Table S2, Additional file 4).

All maximum likelihood sequence analyses were conducted using RAxML 8.1.11 [53] on the CIPRES Science Gateway [54]. For the CR1 phylogeny, we used the alignment from ref. [20], excluded grebe-specific CR1 elements, and added the CR1-derived tails of TguSINE1 and PittSINE (alignment length $710 \mathrm{bp}$ ). For the passerine phylogeny, we removed the RE sequences from our presence/absence alignments and concatenated the remaining RE-flanking sequences into a multilocus alignment (Additional file 4; alignment length 22,410 bp).

Zebra finch TE landscapes were generated from RepeatMasker [55] '.align' files after CpG correction as detailed elsewhere [32]. For PittSINE copies and the PittSINE consensus, Kimura 2-parameter pairwise distances were estimated in MEGA6 ([56]; uniform rates among sites, pairwise deletion of gaps/missing data) after exclusion of CpG sites.

\section{Additional files}

Additional file 1: Tables S1-S4. (PDF $312 \mathrm{~kb}$ )

Additional file 2: Majority-rule consensus sequence for PittSINE as reconstructed from our PittSINE-bearing presence/absence patterns. (TXT 155 bytes)

Additional file 3: Figures S1-S2. (PDF $447 \mathrm{~kb}$ )

Additional file 4: Fasta-formatted alignments of all RE presence/absence markers. The presented loci are labeled corresponding to the markers listed in Additional file 1: Table S2. The names of transposed elements correspond to those in Repbase. (TXT 805 kb)

Additional file 5: Fasta-formatted multilocus alignment of concatenated RE-flanking sequences used for generating the phylogenetic tree of Fig. $3 a$. (TXT $660 \mathrm{~kb}$ )

\section{Abbreviations}

CR1: Chicken repeat 1; LINE: Long interspersed element; Mb: Million basepairs; MY: Million years; MYA: Million years ago; RE: Retroposed element; RT: Reverse transcriptase; RTBS: Reverse transcriptase binding site; SINE: Short interspersed element

\section{Acknowledgements}

We thank Tim Pock, Meike Hüdig, and Felix Babatz for help with in-vitro experiments, and Gerald Mayr and Gennady Churakov for helpful discussions. We are grateful to Leanne Wheaton, Simone Schehka (Allwetterzoo Münster), Robert Palmer, Stephanie Hodges, Geoffrey E. Hill, Franziska A. Franke, Sharon Birks (Burke Museum), and Werner Beckmann (LWL-DNA- und Gewebearchiv) for providing blood and tissue samples, and to Jón Baldur Hliðoberg for generating the bird paintings. We thank three anonymous reviewers for their valuable comments. Some of the computations were performed on resources provided by the Swedish National Infrastructure for Computing (SNIC) through Uppsala Multidisciplinary Center for Advanced Computational Science (UPPMAX).

\section{Funding}

This research was funded by the Deutsche Forschungsgemeinschaft (KR3639 to J.O.K. and J.S.).

\section{Availability of data and materials}

All data generated or analyzed during this study are included in this published article and its supplementary information files. All newly generated sequences were deposited in GenBank (accession numbers MG645622-MG645803).

\section{Authors' contributions}

AS, JOK and JS conceived the project. AS designed the study. AS and JS performed in silico experiments. AS and SB performed in vitro experiments. $\mathrm{SB}, \mathrm{LJ}, \mathrm{JB}, \mathrm{JOK}$ and JS contributed reagents, materials and analysis tools. AS analyzed the data and wrote the manuscript. JS, JOK and JB discussed and commented on the data and the manuscript. All authors read and approved the final manuscript.

Ethics approval and consent to participate Not applicable. 


\section{Consent for publication}

Not applicable.

\section{Competing interests}

The authors declare that they have no competing interests.

\section{Publisher's Note}

Springer Nature remains neutral with regard to jurisdictional claims in published maps and institutional affiliations.

\section{Author details}

'Institute of Experimental Pathology (ZMBE), University of Münster, D-48149 Münster, Germany. ${ }^{2}$ Department of Evolutionary Biology (EBC), Uppsala University, SE-75236 Uppsala, Sweden. ${ }^{3}$ South Australian Museum, Adelaide, SA 5000, Australia. ${ }^{4}$ School of Biological Sciences, The University of Adelaide, Adelaide 5005, Australia. ${ }^{5}$ Australian National Wildlife Collection, CSIRO National Research Collections Australia, Canberra, ACT 2601, Australia. ${ }^{6}$ Brandenburg Medical School (MHB), D-16816 Neuruppin, Germany. 'LWL-Museum für Naturkunde, Westfälisches Landesmuseum mit Planetarium, D-48161 Münster, Germany.

Received: 18 September 2017 Accepted: 29 November 2017 Published online: 14 December 2017

\section{References}

1. Sotero-Caio C, Platt RN II, Suh A, Ray DA. Evolution and diversity of transposable elements in vertebrate genomes. Genome Biol Evol. 2017; 9(1):161-77.

2. Ohshima K, Hamada M, Terai Y, Okada N. The $3^{\prime}$ ends of tRNA-derived short interspersed repetitive elements are derived from the $3^{\prime}$ ends of long interspersed repetitive elements. Mol Cell Biol. 1996;16(7):3756-64.

3. Lander ES, Linton LM, Birren B, Nusbaum C, Zody MC, Baldwin J, Devon K, Dewar K, Doyle M, Fitzhugh W, et al. Initial sequencing and analysis of the human genome. Nature. 2001;409(6822):860-921.

4. Hillier LW, Miller W, Birney E, Warren W, Hardison RC, Ponting CP, Bork P, Burt DW, Groenen MA, Delany ME, et al. Sequence and comparative analysis of the chicken genome provide unique perspectives on vertebrate evolution. Nature. 2004;432(7018):695-716.

5. Warren WC, Clayton DF, Ellegren H, Arnold AP, Hillier LW, Künstner A, Searle S, White S, Vilella AJ, Fairley $S$, et al. The genome of a songbird. Nature. 2010;464(7289):757-62

6. Zhang G, Li C, Li Q, Li B, Larkin DM, Lee C, Storz JF, Antunes A, Greenwold MJ, Meredith RW, et al. Comparative genomics reveals insights into avian genome evolution and adaptation. Science. 2014;346(6215):1311-20.

7. Kapusta A, Suh A. Evolution of bird genomes-a transposon's-eye view. Ann N Y Acad Sci. 2017:1389:164-85.

8. Ray DA, Xing J, Salem A-H, Batzer MA. SINEs of a nearly perfect character. Syst Biol. 2006;55(6):928-35.

9. Shedlock AM, Takahashi K, Okada N. SINEs of speciation: tracking lineages with retroposons. Trends Ecol Evol. 2004;19(10):545-53.

10. Kaiser VB, van Tuinen M, Ellegren $\mathrm{H}$. Insertion events of CR1 retrotransposable elements elucidate the phylogenetic branching order in galliform birds. Mol Biol Evol. 2007;24(1):338-47.

11. Kriegs JO, Matzke A, Churakov G, Kuritzin A, Mayr G, Brosius J, Schmitz J. Waves of genomic hitchhikers shed light on the evolution of gamebirds (Aves: Galliformes). BMC Evol Biol. 2007;7:190.

12. Liu Z, He L, Yuan H, Yue B, Li J. CR1 retroposons provide a new insight into the phylogeny of Phasianidae species (Aves: Galliformes). Gene. 2012;502(2):125-32.

13. Suh A, Paus M, Kiefmann M, Churakov G, Franke FA, Brosius J, Kriegs JO, Schmitz J. Mesozoic retroposons reveal parrots as the closest living relatives of passerine birds. Nat Commun. 2011;2:443.

14. Suh $A$, Smeds $L$, Ellegren $H$. The dynamics of incomplete lineage sorting across the ancient adaptive radiation of neoavian birds. PLoS Biol. 2015; 13(8):e1002224.

15. Matzke A, Churakov G, Berkes P, Arms EM, Kelsey D, Brosius J, Kriegs JO, Schmitz J. Retroposon insertion patterns of neoavian birds: strong evidence for an extensive incomplete lineage sorting era. Mol Biol Evol. 2012;29(6):1497-501.
16. Baker AJ, Haddrath O, McPherson JD, Cloutier A. Genomic support for a moa-tinamou clade and adaptive morphological convergence in flightless ratites. Mol Biol Evol. 2014;31(7):1686-96.

17. Haddrath O, Baker AJ. Multiple nuclear genes and retroposons support vicariance and dispersal of the palaeognaths, and an early cretaceous origin of modern birds. Proc R Soc B. 2012;279(1747):4617-25.

18. Watanabe M, Nikaido M, Tsuda TT, Inoko H, Mindell DP, Murata K, Okada N: The rise and fall of the CR1 subfamily in the lineage leading to penguins. Gene 2006, 365(0):57-66.

19. Kuramoto T, Nishihara H, Watanabe M, Okada N. Determining the position of storks on the phylogenetic tree of waterbirds by retroposon insertion analysis. Genome Biol Evol. 2015;7(12):3180-9.

20. Suh A, Kriegs JO, Donnellan S, Brosius J, Schmitz J. A universal method for the study of CR1 retroposons in nonmodel bird genomes. Mol Biol Evol. 2012:29:2899-903.

21. St. John J, Cotter J-P, Quinn TW. A recent chicken repeat 1 retrotransposition confirms the Coscoroba-Cape barren goose clade. Mol Phylogenet Evol. 2005;37(1):83-90.

22. Suh A, Witt CC, Menger J, Sadanandan KR, Podsiadlowski L, Gerth M, Weigert A, McGuire JA, Mudge J, Edwards SV, et al. Ancient horizontal transfers of retrotransposons between birds and ancestors of human pathogenic nematodes. Nat Commun. 2016;7:11396.

23. Barker FK, Cibois A, Schikler P, Feinstein J, Cracraft J. Phylogeny and diversification of the largest avian radiation. Proc Natl Acad Sci U S A. 2004; 101(30):11040-5.

24. Treplin S, Tiedemann R. Specific chicken repeat 1 (CR1) retrotransposon insertion suggests phylogenetic affinity of rockfowls (genus Picathartes) to crows and ravens (Corvidae). Mol Phylogenet Evol. 2007;43(1):328-37.

25. Selvatti AP, Gonzaga LP, Russo CA. A Paleogene origin for crown passerines and the diversification of the oscines in the new world. Mol Phylogenet Evol. 2015;88:1-15.

26. Moyle RG, Oliveros CH, Andersen MJ, Hosner PA, Benz BW, Manthey JD, Travers SL, Brown RM, Faircloth BC. Tectonic collision and uplift of Wallacea triggered the global songbird radiation. Nat Commun. 2016;7:12709.

27. Suh A, Kriegs JO, Brosius J, Schmitz J. Retroposon insertions and the chronology of avian sex chromosome evolution. Mol Biol Evol. 2011;28:2993-7.

28. Benson DA, Cavanaugh M, Clark K, Karsch-Mizrachi I, Lipman DJ, Ostell J, Sayers EW. GenBank. Nucleic Acids Res. 2013;41(D1):D36-42.

29. Irestedt M, Ohlson Jl, Zuccon D, Källersjö M, Ericson PGP. Nuclear DNA from old collections of avian study skins reveals the evolutionary history of the old world suboscines (Aves, Passeriformes). Zool Scripta. 2006;35(6):567-80.

30. Kaukinen J, Varvio S-L. Artiodactyl retroposons: association with microsatellites and use in SINEmorph detection by PCR. Nucleic Acids Res. 1992;20(12):2955-8.

31. Suh $A$. The specific requirements for $C R 1$ retrotransposition explain the scarcity of retrogenes in birds. J Mol Evol. 2015;81:18-20.

32. Suh A, Churakov G, Ramakodi MP, Platt RN II, Jurka J, Kojima KK, Caballero J, Smit A, Vliet K, Hoffmann FG, et al. Multiple lineages of ancient CR1 retroposons shaped the early genome evolution of amniotes. Genome Biol Evol. 2015;7(1):205-17.

33. Kajikawa M, Okada N. LINEs mobilize SINEs in the eel through a shared 3' sequence. Cell. 2002;111(3):433-44.

34. Cracraft J. Avian higher-level relationships and classification: Passeriforms. In: The Howard and Moore Complete Checklist of the Birds of the World 4th Edition. Edited by Dickinson EC, Christidis L, vol. 2. Eastbourne, U.K.: Aves Press; 2014: xvii-xlv.

35. Waddell PJ, Kishino H, Ota R. A phylogenetic foundation for comparative mammalian genomics. Genome Inform. 2001;12:141-54.

36. Kuritzin A, Kischka T, Schmitz J, Churakov G. Incomplete lineage sorting and hybridization statistics for large-scale retroposon insertion data. PLoS Comput Biol. 2016;12(3):e1004812.

37. Mayr G, Manegold A. The oldest European fossil songbird from the early Oligocene of Germany. Naturwissenschaften. 2004;91(4):173-7.

38. Ericson PG, Christidis L, Cooper A, Irestedt M, Jackson J, Johansson US, Norman JA. A Gondwanan origin of passerine birds supported by DNA sequences of the endemic New Zealand wrens. Proc R Soc B. 2002; 269(1488):235-41.

39. Barker FK, Barrowclough GF, Groth JG. A phylogenetic hypothesis for passerine birds: taxonomic and biogeographic implications of an analysis of nuclear DNA sequence data. Proc R Soc Lond Ser B Biol Sci. 2002;269(1488):295-308. 
40. Ericson P, Klopfstein S, Irestedt M, Nguyen J, Nylander J. Dating the diversification of the major lineages of Passeriformes (Aves). BMC Evol Biol. 2014;14(1):8

41. Suh A. The phylogenomic forest of bird trees contains a hard polytomy at the root of Neoaves. Zool Scripta. 2016;45(S1):50-62.

42. Han K-L, Braun EL, Kimball RT, Reddy S, Bowie RCK, Braun MJ, Chojnowski JL, Hackett SJ, Harshman J, Huddleston CJ, et al. Are transposable element insertions homoplasy free?: an examination using the avian tree of life. Syst Biol. 2011;60(3):375-86.

43. Manegold A, Mayr G, Mourer-Chauviré C. Miocene songbirds and the composition of the European passeriform avifauna. Auk. 2004;121(4):1155-60.

44. Gilbert N, Labuda D. Evolutionary inventions and continuity of CORE-SINEs in mammals. J Mol Biol. 2000;298(3):365-77.

45. Buzdin A, Ustyugova S, Gogvadze E, Vinogradova T, Lebedev Y, Sverdlov E. A new family of chimeric retrotranscripts formed by a full copy of U6 small nuclear RNA fused to the 3' terminus of L1. Genomics. 2002;80(4):402-6.

46. Nishihara H, Plazzi F, Passamonti M, Okada N. MetaSINEs: broad distribution of a novel SINE superfamily in animals. Genome Biol Evol. 2016;8(3):528-39.

47. Brosius J. Genomes were forged by massive bombardments with retroelements and retrosequences. Genetica. 1999;107(1):209-38.

48. Ohshima K, Okada N. SINEs and LINEs: symbionts of eukaryotic genomes with a common tail. Cytogenet Genome Res. 2005:110(1-4):475-90.

49. Nishihara H, Smit AFA, Okada N. Functional noncoding sequences derived from SINEs in the mammalian genome. Genome Res. 2006;16(7):864-74.

50. Fujita P, Rhead B, Zweig A, Hinrichs A, Karolchik D, Cline MS, Goldman M, Barber G, Clawson H, Coelho ADM, Dreszer TR, Giardine BM, Harte RA, HillmanJackson J, Hsu F, Kirkup V, Kuhn RM, Learned K, Li CH, Meyer LR, Pohl A, Raney BJ, Rosenbloom KR, Smith KE, Haussler D, Kent WJ. The UCSC genome browser database: update 2011. Nucleic Acids Res. 2011;39:D876-82.

51. Katoh K, Toh H. Recent developments in the MAFFT multiple sequence alignment program. Brief Bioinform. 2008;9:286-98.

52. Poelstra JW, Vijay N, Bossu CM, Lantz H, Ryll B, Müller I, Baglione V, Unneberg P, Wikelski M, Grabherr MG, et al. The genomic landscape underlying phenotypic integrity in the face of gene flow in crows. Science. 2014:344(6190):1410-4.

53. Stamatakis A, Hoover P, Rougemont J. A rapid bootstrap algorithm for the RAxML web servers. Syst Biol. 2008;75:758-71.

54. Miller MA, Pfeiffer W, Schwartz T: Creating the CIPRES Science Gateway for inference of large phylogenetic trees. Proceedings of the Gateway Computing Environments Workshop (GCE). 2010:1-8.

55. Smit A, Hubley R, Green P. RepeatMasker Open-3.3.0. http://www. repeatmasker.org. 1996-2010.

56. Tamura K, Stecher G, Peterson D, Filipski A, Kumar S. MEGA6: molecular evolutionary genetics analysis version 6.0. Mol Biol Evol. 2013;30(12):2725-9.

57. Zuker M. Mfold web server for nucleic acid folding and hybridization prediction. Nucleic Acids Res. 2003;31(13):3406-15.

58. Jurka J, Kapitonov W, Pavlicek A, Klonowski P, Kohany O, Walichiewicz J. Repbase update, a database of eukaryotic repetitive elements. Cytogenet Genome Res. 2005;110(1-4):462-7.

\section{Submit your next manuscript to BioMed Central and we will help you at every step:}

- We accept pre-submission inquiries

- Our selector tool helps you to find the most relevant journal

- We provide round the clock customer support

- Convenient online submission

- Thorough peer review

- Inclusion in PubMed and all major indexing services

- Maximum visibility for your research

Submit your manuscript at www.biomedcentral.com/submit 